\title{
Construction of axial chirality via asymmetric radical trapping by cobalt under visible light
}

Wen-Jing Xiao ( $\Delta$ wxiao@mail.ccnu.edu.cn )

Central China Normal University https://orcid.org/0000-0002-9318-6021

Xuan Jiang

Central China Normal University

Wei Xiong

Central China Normal University

Shuang Deng

Wuhan University

Fu-Dong Lu

Central China Normal University

Yue Jia

Central China Normal University

Qian Yang

Central China Normal University

Li-Yuan Xue

Central China Normal University

Xiaotian Qi

Institute for Advanced Studies (IAS), College of Chemistry and Molecular Sciences, Wuhan University https://orcid.org/0000-0001-5420-5958

Jon A. Tunge

University of Kansas

Liang-Qiu Lu

Central China Normal University https://orcid.org/0000-0003-2177-4729

Article

Keywords:

Posted Date: February 17th, 2022

DOI: https://doi.org/10.21203/rs.3.rs-1268664/v1

License: (9) This work is licensed under a Creative Commons Attribution 4.0 International License.

Read Full License 
Version of Record: A version of this preprint was published at Nature Catalysis on September 15th, 2022. See the published version at https://doi.org/10.1038/s41929-022-00831-1. 


\title{
Construction of axial chirality via asymmetric radical trapping by cobalt under visible light
}

\author{
Xuan Jiang ${ }^{1}$, Wei Xiong ${ }^{1}$, Shuang Deng ${ }^{2}$, Fu-Dong Lu ${ }^{1}$, Yue Jia ${ }^{1}$, Qian Yang ${ }^{1}$, Li-Yuan \\ Xue $^{1}$, Xiaotian $\mathrm{Qi}^{2}$, Jon A. Tunge ${ }^{3}$, Liang-Qiu Lu ${ }^{1,4, \square} \&$ Wen-Jing Xiao ${ }^{1,5, \square}$ \\ ${ }^{1}$ CCNU-uOttawa Joint Research Centre, Key Laboratory of Pesticides \& Chemical Biology Ministry of \\ Education, College of Chemistry, Central China Normal University, 152 Luoyu Road, Wuhan 430079, China \\ ${ }^{2}$ Engineering Research Center of Organosilicon Compounds \& Materials, Ministry of Education, College of \\ Chemistry and Molecular Sciences, Wuhan University, Wuhan 430072, China \\ ${ }^{3}$ Department of Chemistry, The University of Kansas, 1567 Irving Hill Rd., Lawrence KS 66045, USA \\ ${ }^{4}$ State Key Laboratory for Oxo Synthesis and Selective Oxidation, Lanzhou Institute of Chemical Physics \\ (LICP), Chinese Academy of Sciences, Lanzhou 730000, China \\ ${ }^{5}$ State Key Laboratory of Applied Organic Chemistry, Lanzhou University, Lanzhou 730000, China \\ *These authors contributed equally to this work \\ $\square_{\text {e-mail: luliangqiu@mail.ccnu.edu.cn; wxiao@ mail.ccnu.edu.cn }}$
}

\begin{abstract}
Metals have been identified as economic and sustainable alternatives to palladium, the frequently used metal in transition-metal-catalyzed cross-couplings. However, cobalt has long stood behind its neighboring elements, nickel and copper, in asymmetric radical couplings, owing to its high catalytic activity in the absence of ligands. Here, we disclose an asymmetric metallaphotoredox catalysis (AMPC) strategy for the dynamic kinetic resolution (DKR) of racemic heterobiaryls, which represents the first example of visible-light-induced, asymmetric radical couplings for the construction of axial chirality. This success can also be extended to the reductive cross-coupling variant featuring on more easily available feedstocks. The keys to these successes are the rational design of a sustainable AMPC system by merging asymmetric cobalt catalysis with organic photoredox catalysis and, perhaps more importantly, the identification of an efficient chiral polydentate ligand.
\end{abstract}

Over the last decade, organic photochemical synthesis that utilizes sustainable, abundant visible light has witnessed a noticeable renaissance ${ }^{1,2}$. Due to the high reactivity of open-shelled intermediates, asymmetric photochemical transformations under visible-light ${ }^{3}$ are believed to be challenging. Even so, stereocontrol strategies including asymmetric organocatalysis $^{4}$, organometallic catalysis ${ }^{5}$ and enzyme catalysis ${ }^{6}$ have been gradually applied to this area. As highlighted in Fig. 1a, the emerging dual catalysis strategy has achieved considerable success in the construction of chiral carbon stereocenters ${ }^{7-16}$; however, its application in the construction of axially chiral molecules is in its infancy ${ }^{17}$. To the best of our knowledge, there has only been one chiral bifunctional catalyst system which was recently reported by Bach, who disclosed a catalytic deracemization of allene lactams via an energy transfer (EnT) mechanism under visible light (as shown in Fig. 1b) ${ }^{18}$ and later expanded the system to primary allene amides ${ }^{19}$. In this work, we disclosed two unprecedented radical-pathway DKRs of racemic heterobiaryls through synergistic 
photoredox-cobalt catalysis for constructing significant axially chiral scaffolds.

Cobalt-catalyzed cross-couplings have been well-known as one of the most powerful approaches to forge chemical bonds ${ }^{20}$. However, as an important 3d-metal, cobalt has been much less studied for use in asymmetric radical couplings ${ }^{21-23}$ than its neighbors, nickel ${ }^{24}$ and copper $^{25,26}$. Recently, metallaphotoredox-catalyzed radical cross-couplings that merge photoredox catalysis and 3d-metal catalysis have flourished ${ }^{27}$ and again, cobalt $^{28}$ stands $^{2}$ behind nickel and copper in its utilization. One main reason might be that the high catalytic activity of cobalt salts themselves in the absence of additional ligand hinders chiral ligand-promoted asymmetric couplings. Because of our research interests in asymmetric photochemical transformations under visible light ${ }^{29-31}$, we recently questioned whether chiral polydentate ligands could increase the possibility of coordination with cobalt. More importantly, compared with the extensively used $\mathrm{Cu}(\mathrm{I})$ and $\mathrm{Ni}(0)$ which have 10 outermost electrons (d10), the relatively empty 3d-orbital of $\mathrm{Co}(\mathrm{I})$ (8 outermost electrons, d8) would allow polydentate binding to not affect the catalytic performance in oxidative addition; again, compared with the well-known planar coordination structures of $\mathrm{Pd}(\mathrm{II})$ and $\mathrm{Ni}$ (II), the
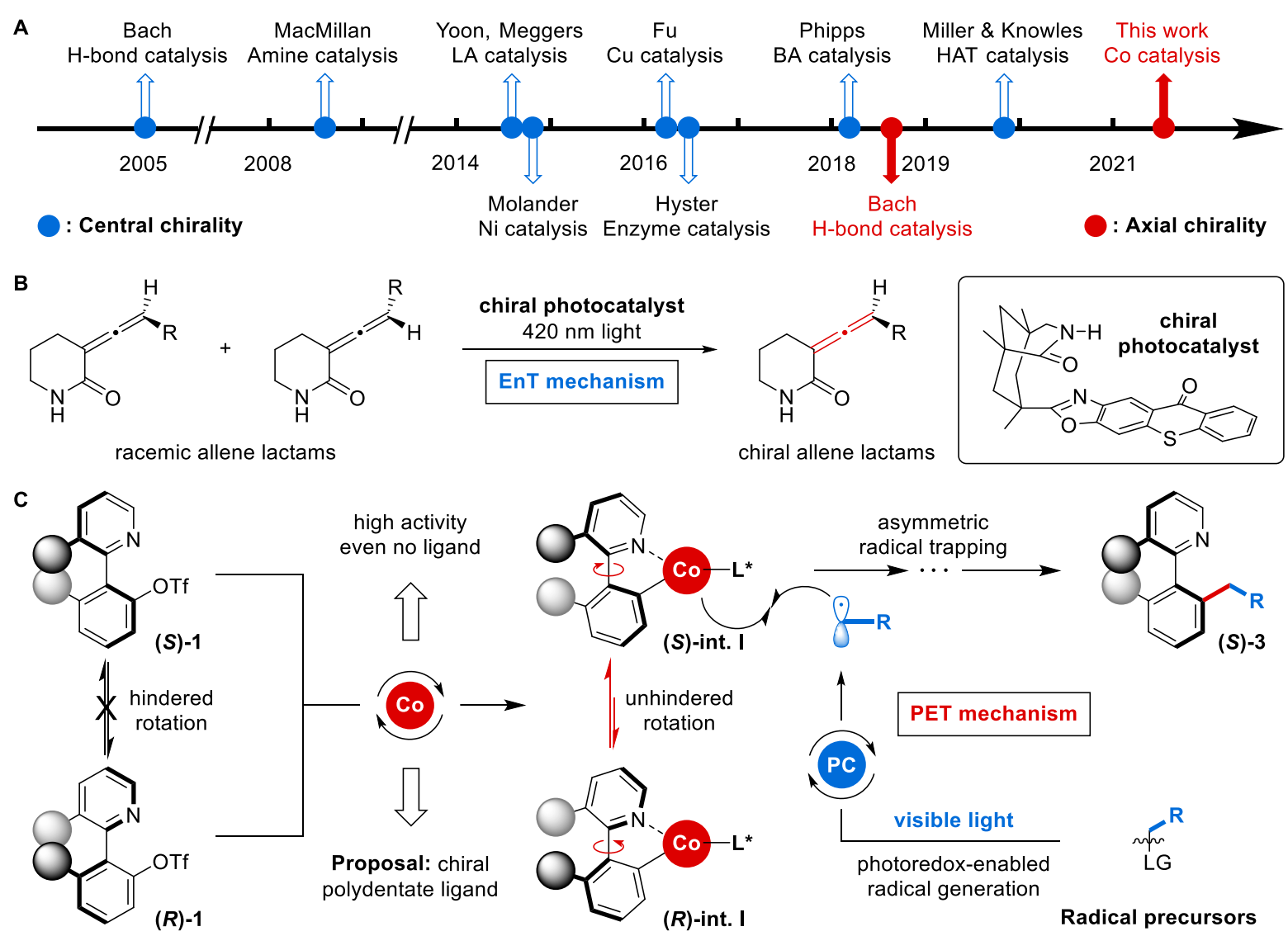

hiral allene lactams

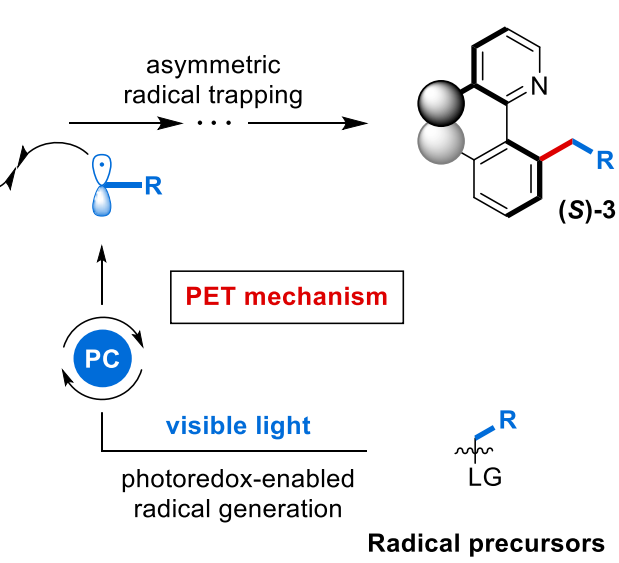

Previous studies:

- precious metal catalysts (Pd, Rh, Ir)

- even-electron reaction mechanism

- not applicable to alkyl nucleophiles
Advantagies in this study:
- sustainable, less toxic $3 \mathrm{~d}$ metal \& metal-free photosensitizer
- single-electron reaction mechanism under visible light
- avoiding air- and moisture-sensitive organometallic reagents

Fig. 1 | Design blueprint for the AMPC-enabled DKR of racemic heterobiaryls. (a) A brief history of asymmetric photocatalysis. (b) Bach's deracemization reactions for synthesizing chiral allene lactams. (c) Proposal of this work: construction of axially chiral heterobiaryls through synergistic cobalt/photoredox catalysis. PET: photoinduced electron transfer. LG: leaving group. 
octahedral coordination of Co(III) would be more favored for asymmetric induction by the chiral polydentate ligand. Based on this assumption, and inspired by the elegant work on chelation-directed DKRs of racemic heterobiaryls ${ }^{32-35}$, herein, we apply the radical-pathway DKR of easily available, configuration-stable heterobiaryl triflates as the platform to develop new AMPC systems by merging organic photoredox catalysis and asymmetric cobalt catalysis.

\section{Design plan}

As illustrated in Fig. 1c, we assumed that oxidative addition of low-valent cobalt would afford cationic cyclic cobalt intermediates $(\boldsymbol{R})$-int. I and $(\boldsymbol{S})$-int. I. As a basis of this proposal, the coordination of cobalt with the basic nitrogen atom in the heterobiaryl scaffold would keep the two steric groups far from each other compared with their positions in starting materials $\mathbf{1}$, and the conversion of $(\boldsymbol{R})$-int. I into $(\boldsymbol{S})$-int. I via axial rotation would be more

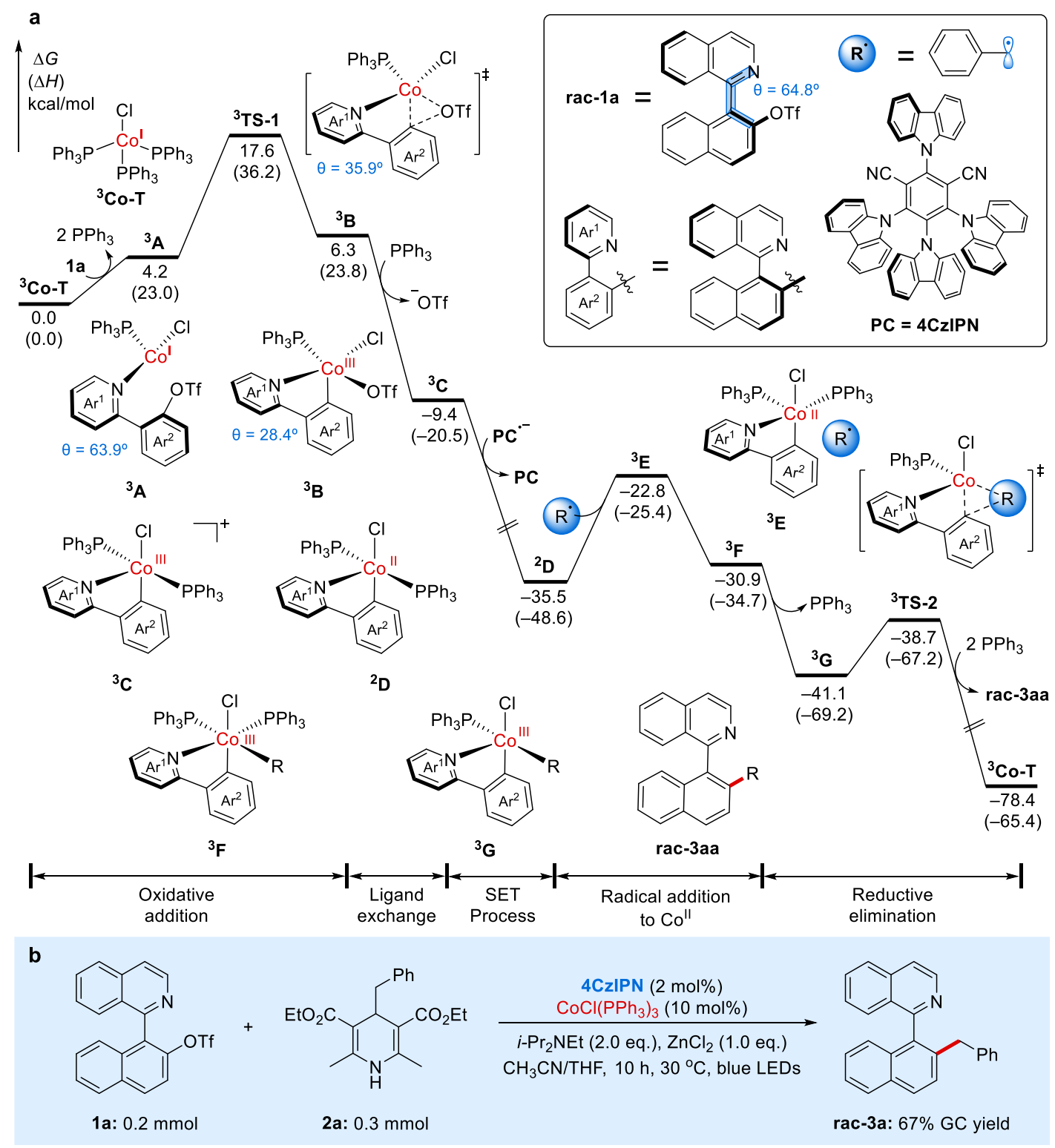

Fig. 2 | Probe the feasibility of the reactivity of cobalt/photoredox-catalyzed racial coupling. (a) Study on the reaction profile via DFT calculation. (b) A preliminary trial on cobalt/photoredox-catalyzed radical coupling. 
easily induced by chiral ligands. Theoretically, the smaller atom radii of $3 \mathrm{~d}$-metals would make the rotation process more favorable than that with $4 \mathrm{~d}$ metals. Next, asymmetric trapping of photoredox-generated radicals by the chiral cobalt intermediate $(\boldsymbol{S})$-int. I would afford the desired axially chiral heterobiaryl product through reductive elimination. According to this reaction scenario, quick axial rotation and stereo-favored radical trapping are critical for both high enantioselectivity and efficiency. If successful, this 3d-metal cobalt-involved, metallaphotoredox-catalyzed radical DKR will add a new and unique member to the arsenal of techniques for chiral heterobiaryl construction ${ }^{36}$, which is difficult through precious-metal-catalyzed two-electron processes. Additionally, these radical DKR processes will be highly valued because of their avoidance of air- and moisture-sensitive organometallic reagents, thus increasing the practicality and functional-group compatibility of the methodology. Despite these promising advantages, the underlying challenges include 1) the compatibility of cobalt catalysis with photoredox catalysis, 2) the satisfactory chemo- and enantioselectivity of asymmetric radical coupling, and 3) the potential for reductive dehalogenation (triflates are a kind of halogenoid widely used in transition-metal-catalyzed cross-couplings) which, according to preceding literature, would compete with the designed process through aryl-cobalt decomposition ${ }^{37}$. On the other hand, to date, successful cobalt-catalyzed asymmetric radical cross-couplings are rare, perhaps because less efficient catalytic systems have been utilized.

To examine the feasibility of the required reactivity of our hypothesis, we initially theoretically investigated the cobalt/photoredox-catalyzed radical cross-coupling pathway using heterobiaryl 1a and DHP 2a as the model reactants. According to the preceding literature on cobalt catalysis and photoredox catalysis, the newly designed coupling reaction would include $\mathrm{C}-\mathrm{O}$ oxidative addition to $\mathrm{Co}(\mathrm{I})$, ligand exchange, single-electron reduction of $\mathrm{Co}(\mathrm{III})$ to $\mathrm{Co}(\mathrm{II})$, benzyl radical addition to $\mathrm{Co}(\mathrm{II})$ and $\mathrm{C}-\mathrm{C}$ reductive elimination (as shown in Fig. 2a). Our density functional theory (DFT) results suggest that formation of triplet $\mathrm{Co}(\mathrm{III})$ intermediate ${ }^{\mathbf{3}} \mathbf{B}$ through $\mathrm{C}-\mathrm{O}$ oxidative addition to triplex $\mathrm{Co}(\mathrm{I})$ complex $\left({ }^{\mathbf{3}} \mathbf{T S}-\mathbf{1}\right)$ is the rate-determining step and requires an activation free energy of only $17.6 \mathrm{kcal} / \mathrm{mol}$. The ligand exchange between $\mathrm{PPh}_{3}$ and $\mathrm{TfO}^{-}$can form a more stable $\mathrm{Co}(\mathrm{III})$ intermediate ${ }^{3} \mathrm{C}$. Notably, single-electron transfer (SET) between ${ }^{3} \mathbf{C}$ and $\mathbf{P C}^{\bullet-}$ is exergonic by $26.1 \mathrm{kcal} / \mathrm{mol}$, indicating that the single-electron reduction of $\mathrm{Co}(\mathrm{III})$ to $\mathrm{Co}(\mathrm{II})$ is thermodynamically favored. Although the transition state of benzyl radical addition to doublet $\mathrm{Co}$ (II) complex ${ }^{2} \mathbf{D}$ is not obtained, computational results show that the generation of $\mathrm{Co}$ (III) intermediate ${ }^{\mathbf{3}} \mathbf{G}$ after radical addition $\left({ }^{2} \mathbf{D} \rightarrow{ }^{3} \mathbf{F}\right)$ followed by $\mathrm{PPh}_{3}$ dissociation $\left({ }^{3} \mathbf{F} \rightarrow{ }^{\mathbf{3}} \mathbf{G}\right)$ is exergonic by 5.6 $\mathrm{kcal} / \mathrm{mol}$, which indicates that the formation of ${ }^{\mathbf{3}} \mathbf{G}$ is a feasible process. Finally, the formation of coupling product rac-3a through the $\mathrm{C}-\mathrm{C}$ reductive elimination transition state ${ }^{3} \mathbf{T S}-\mathbf{2}$ is highly exergonic by $78.4 \mathrm{kcal} / \mathrm{mol}$ and has an energy barrier of only $2.4 \mathrm{kcal} / \mathrm{mol}$. Therefore, the DFT calculations suggested that the design radical coupling is theoretically feasible by merging cobalt catalysis and photoredox catalysis. To verify this theoretical study, we performed a radical coupling reaction with heterobiaryl 1a and DHP 2a in the presence of the cobalt catalyst $\mathrm{Co}(\mathrm{I})\left(\mathrm{PPh}_{3}\right)_{3} \mathrm{Cl}$ and an organic photocatalyst under the irradiation of blue LEDs. Indeed, the desired coupling product rac-3a was facilely obtained in $67 \%$ yield (as shown in Fig. 2b). Furthermore, computational studies also revealed that along with the $\mathrm{C}-\mathrm{O}$ oxidative addition to $\operatorname{Co}(\mathrm{I})$, the dihedral angle $(\theta)$ in $\mathbf{1 a}$ formed by the planes of the two 
aromatic rings significantly decreases from $64.8^{\circ}$ to $28.4^{\circ}$ (intermediate $\mathbf{B}$, as shown in Fig. 2a). This prominent geometric change can diminish the steric clash between two aromatic rings amid the $\mathrm{C}-\mathrm{C}$ axial rotation, which paves the way for a rational design of the dynamic kinetic resolution of racemic heterobiaryls.

\section{Experimental results}

Condition optimizations for the AESC-enabled radical DKR. Encouraged by the above success, we turned our attention to experimentally studying the asymmetric process. The metallaphotoredox-catalyzed DKR was optimized with respect to the chiral ligands, cobalt salts and other reaction parameters. The results of the ligand effect study are summarized in Fig. 3a. We found that chiral monodentate phosphine ligand L1 failed to induce any enantioselectivity, although the product was obtained in a high yield (90\% yield, 50:50 er). Chiral bidentate bisoxazoline ligands, which showed high efficiency and enantioselectivity in cobalt-catalyzed asymmetric radical couplings of $\alpha$-bromocarbonyls ${ }^{21}$, do not give satisfactory results here. Among them, the best ligand was $\mathbf{L} 2$ that gave product in $29 \%$ yield and 68:32 er (see more results in the Supplementary Information). Later, chiral polydentate ligands, P,N,N-ligands $\mathbf{L 3}$ and $\mathbf{L} 4$ were examined achieving very low enantioselectivities in both cases (L3: 69\% yield, 53: 47 er; L4: 80\% yield, 63:37 er). Next, we screened other electron-rich chiral P-containing ligands L5-L8, which were exploited by Zhang's group usually as chiral bifunctional organocatalysts (see more results in the Supplementary Information $)^{38}$. To our delight, chiral biphosphine ligand $\mathbf{L 8}$ was determined to be the best ligand, resulting in 90\% yield and 85:15 er. To understand the function of the sulfinyl amine unit, two additional ligands $\mathbf{L 9}$ and $\mathbf{L 1 0}$ were tested. The results show that the chiral sulfinyl amine unit plays an important role in both reaction efficiency and good stereocontrol (L8 and L9), and we speculate that this amide might not function as a chiral anionic ligand like Liu's catalyst systems with ligand $\mathbf{L 4}$ because the difference in enantioselectivity was not obvious at $30{ }^{\circ} \mathrm{C}(\mathbf{L 8} \text { v.s } \mathbf{~ L 9})^{22}$. Later, we found that increasing the reaction temperature and using 90 $\mathrm{W}$ blue light can further improve the enantioselectivity, while retaining high yields (Fig. 3b, entries 1-4). Next, a series of cobalt catalyst precursors were evaluated (Fig. 3b, entries 4-9), and $\mathrm{CoCl}_{2}$ gave a slightly better result (Fig. 3b, entry 6: 93\% yield and 96:4 er). Finally, after further optimizing the amount of each component of the reaction (see the details in the Supplementary Information), we confirmed that the combination of $\mathrm{CoCl}_{2}(5 \mathrm{~mol} \%)$, chiral ligand $\mathbf{L 8}(10 \mathrm{~mol} \%), i-\operatorname{Pr}_{2} \mathrm{NEt}$ (1.0 eq.) and $\mathrm{ZnCl}_{2}(20 \mathrm{~mol} \%)$ was the optimal conditions for this visible-light-induced radical DKR process, affording the desired 3a in 92\% isolated yield with 96:4 er (Fig. 3b, entry 10). The omitting of $\mathrm{ZnCl}_{2}$ resulted in a slightly eroded yield and enantioselectivity, but the reason is not unclear at the current stage (Fig. 3b, entry 11; see Table S3 in the Supplementary Information for more results on the study of $\mathrm{ZnCl}_{2}$ effect). To exclude the potential effect of the impurities, a higher-purity $\mathrm{CoCl}_{2}(99.998 \%)$ was used and the same results as the $\mathrm{CoCl}_{2}$ with $98 \%$ purity were observed (entry 12 vs. 10). Moreover, as a comparison, other $3 \mathrm{~d}$-metals including iron, copper and nickel, $4 \mathrm{~d}$ metals including ruthenium, rhodium and palladium, and $5 \mathrm{~d}$ metal iridium were examined under the optimized conditions (Fig. 3b, entries 13-16). We found that all of them failed to generate the desired coupling product 3a except nickel salts, which resulted in a high yield but in a racemic form. These results further confirmed the unique catalytic performance of cobalt in this AMPC-enabled DKR process under visible light. Perhaps more significantly, the success of 
chiral polydentate ligand $\mathbf{L 8}$ experimentally verified our working hypothesis based on the fewer 3d-electrons of $\mathrm{Co}(\mathrm{I})$ and the octahedral coordination of $\mathrm{Co}(\mathrm{III})$.

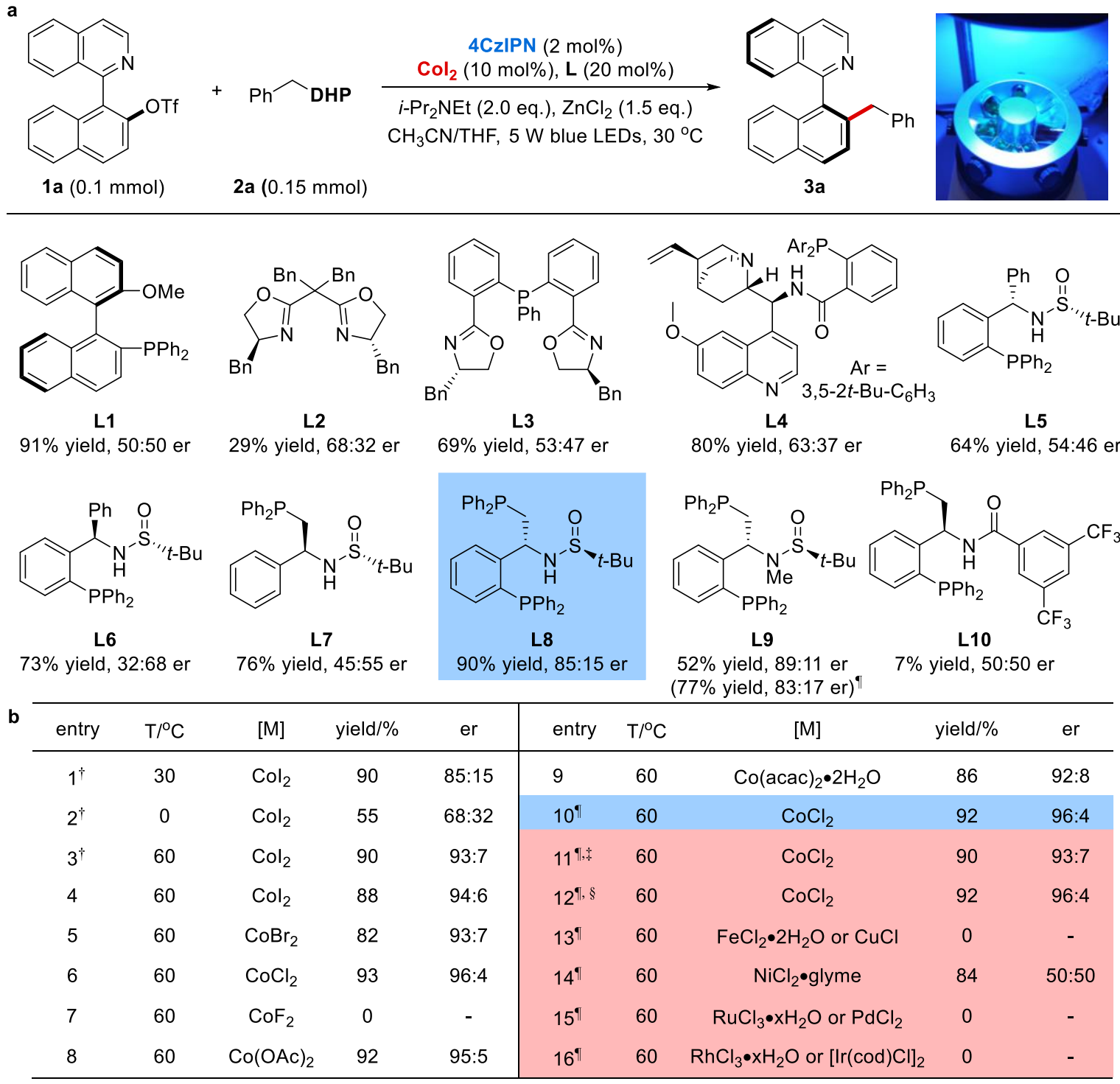

Fig. 3 | Condition optimization for the AMPC-enabled DKR reaction. (a) Chiral ligands evaluated in the model reaction. (b) Effects of other reaction parameters including temperature, light sources, cobalt salts and additives. Unless indicated, all the reactions were performed with $90 \mathrm{~W}$ blue light. ${ }^{\dagger} 5 \mathrm{~W}$ blue LEDs. ${ }^{8} \mathrm{CoCl}_{2}$

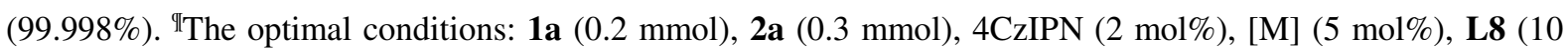

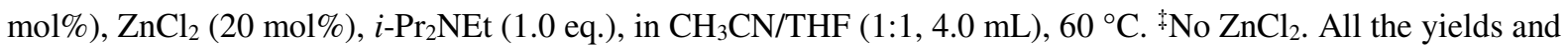
er values were determined by GC and chiral HPLC analysis. cod: cyclooctadiene; er: enantiomer ratio.

Substrate scope for AMPC-enabled radical DKR. Under the optimal reaction conditions, we examined the generality of this radical DKR process. As highlighted in Fig. 4, a variety of DHP reagents were suitable for this transformation. When benzyl-substituted DHPs were used, variations in the electronic character and substitution manner on the benzene ring were tolerant, affording the target products in good yields and high enantioselectivities (3b-3h: 70-98\% yield, up to 97.5:2.5 er). Among them, the structure of product $\mathbf{3 g}$ was unambiguously confirmed through single-crystal X-ray diffraction analysis. 


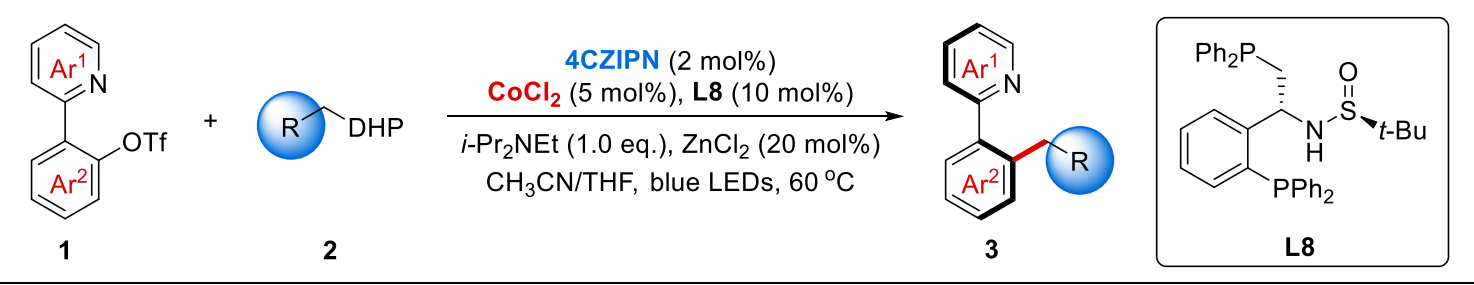

$3 b(\mathrm{R}=4-\mathrm{F}): 95 \%$ yield, $96: 4$ er
$\mathbf{3 c}(\mathrm{R}=4-\mathrm{Cl}): 99 \%$ yield, $96.5: 3.5$ er
$\mathbf{3 d}(\mathrm{R}=4-\mathrm{Me}): 73 \%$ yield, $95: 5$ er
$\mathbf{3 e}(\mathrm{R}=4-\mathrm{Ph}): 70 \%$ yield, $90.5: 9.5$ er
$\mathbf{3 f}(\mathrm{R}=3-\mathrm{Me}): 77 \%$ yield, $95.5: 4.5$ er
$\mathbf{3 g}(\mathrm{R}=3,4-2 \mathrm{MeO}): 98 \%$ yield, $97.5: 2.5$ er

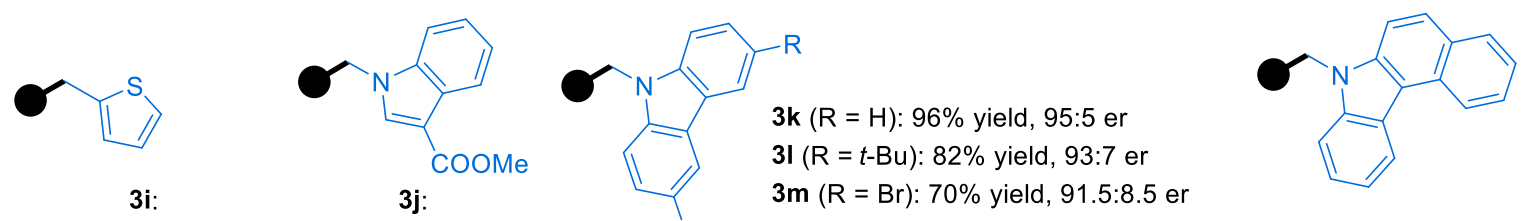

95\% yield, $92.5: 7.5$ er $78 \%$ yield, $94: 6$ er $\quad R \quad$ 3n $(R=C l): 65 \%$ yield, $92.5: 7.5$ er

3o: $99 \%$ yield, $90: 10$ er

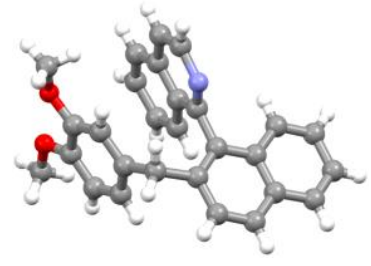

X-ray structure of 3g(CCDC 2124717)<smiles>[R]c1cnc(-c2c(C[Al])ccc3ccccc23)c2ccccc12</smiles><smiles>[R]c1cccc2c(-c3cccc4ccccc34)ccnc12</smiles>

3u $(\mathrm{R}=\mathrm{F})$ : $96 \%$ yield, $91: 9 \mathrm{er}$ 3v $(\mathrm{R}=\mathrm{Cl}): 93 \%$ yield, $97.5: 2.5$ er

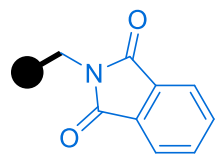
3p:
$99 \%$ yield, $96.5 \cdot 3.5$ er

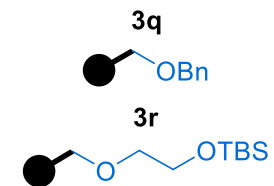

3q: $84 \%$ yield, $96: 4$ er $3 r$ : $74 \%$ yield, $92: 8$ er

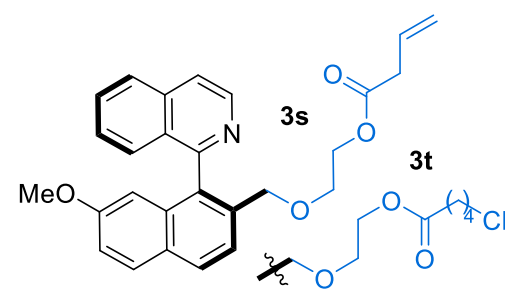

3s: $82 \%$ yield, $94.5: 5.5$ er 3t: $68 \%$ yield, $94.5: 5.5$ er<smiles>[Z]Cc1ccc2ccccc2c1-c1nccc2c([R])cccc12</smiles>

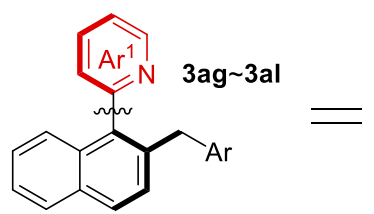<smiles>[R]c1cnc(C(C)(C)C)c(C)c1</smiles>
$3 \mathbf{w}(\mathrm{R}=\mathrm{CN})$ : $94 \%$ yield, $95: 5$ e.r. 3x $(\mathrm{R}=\mathrm{CHO})$ : $72 \%$ yield, $94.5: 5.5$ er 3y $(\mathrm{R}=\mathrm{Br})$ : $93 \%$ yield, $96.5: 3.5$ er $\mathbf{3 z}(\mathrm{R}=\mathrm{Cl})$ : $96 \%$ yield, $95.5: 4.5$ er 3aa $(\mathrm{R}=\mathrm{OMe})$ :

$89 \%$ yield, $96.5: 3.5 \mathrm{er}$

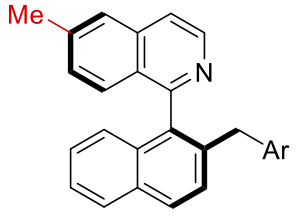

3ab: 93\% yield, $97: 3 \mathrm{er}$<smiles>[Al]Cc1ccc2ccccc2c1-c1nccc2ccc(Br)cc12</smiles>

3ac: $78 \%$ yield, $97: 3$ er 3ad $(\mathrm{R}=\mathrm{Br}): 86 \%$ yield, 94.5:5.5 er 3ae $(R=C l): 71 \%$ yield, $92.5: 7.5$ er 3af $(R=M e O): 97 \%$ yield, $96.5: 3.5$ er 3ag $(R=H): 96 \%$ yield, 95.5:4.5 er

3ah $(R=F): 96 \%$ yield, $97: 3$ er 3ai $(R=C N): 99 \%$ yield, $98.5: 1.5$ er $96.5: 3.5$ er<smiles>CC(C)(C)c1ncccc1CC#N</smiles><smiles>Cc1nccnc1C(C)(C)C</smiles><smiles>COc1ccc2ccc(CBr)c(-c3nccc4ccccc34)c2c1</smiles>

3am:<smiles>COc1cc(CBr)c(-c2nccc3ccccc23)c2ccccc12</smiles>

3 an:
$96 \%$ yield, $97: 3$ er<smiles>N#Cc1ccc2c(-c3nccc4ccccc34)c(CCBr)ccc2c1</smiles>

3ao:
99\% yield, 96.5:3.5 er<smiles>[Al]Cc1ccc2cc(Br)ccc2c1-c1nccc2ccccc12</smiles>

3ap:
97\% yield, $96: 4$ er<smiles>Cc1cccc(C[Al])c1-c1nccc2ccccc12</smiles>

3aq:

Fig. 4 | Substrate scope of the AMPC-enabled radical DKR. All reactions were performed under the optimal conditions (see entry 10 in Fig. 3b). All the yields are isolated yields, and all er values were determined by chiral HPLC analysis. $\mathrm{Ar}=3,4-2 \mathrm{MeO}-\mathrm{C}_{6} \mathrm{H}_{3}$. 
Moreover, DHP reagents containing heteroaryls, such as thiophene, indole and carbazole, can also effectively participate in this transformation, delivering the corresponding chiral heterobiaryl products 3i-3o in 65-99\% yields and up to 95:5 er. In addition to benzyl radicals, asymmetric radical coupling reactions with DHP reagents generating heteroatom-stabilized radicals $(\mathrm{O}$ or $\mathrm{N}$ ) can also proceed well, affording the desired products 3p-3t in 68-99\% yields and up to $96.5: 3.5$ er. To prove the practicality of this methodology, two gram-scale reactions were performed with heterobiaryl $\mathbf{1 a}$ and DHPs $\mathbf{2 k}$ and $\mathbf{2 p}$ under the standard AMPC conditions, affording chiral product $\mathbf{3 k}$ and $\mathbf{3 p}$ in the similar efficiency and enantioselectivity (Fig. 6). Unfortunately, secondary and tertiary carbon radicals are not applicable at the current stage, presumably steric repulsion slowed the reaction such that competitive reductive detriflation of heterobiaryl triflates was observed.

Next, we probed the scope of racemic heterobiaryl components. First, we found that substitution on the isoquinoline ring, i.e., fluoro and chloro at the 4-position and various substituents at the 5 7-positions, are compatible, generating chiral products 3u-3af in 71-97\% yields and up to 97.5:2.5 er. In addition to isoquinoline, heterobiaryl substrates with other nitrogen-containing heterocycles, such as pyridine and pyrazine, were found to be suitable for this radical DKR, generating chiral products 3ag-3al in 53-99\% yields and up to 98.5:1.5 er. Moreover, when substituents were introduced to the 4-, 6- and 7-positons of the naphthalene ring (3am-3aq) or when the naphthalene ring was replaced with a 6-methyl phenyl group (3aq), the reactions also proceeded in high yields and enantioselectivities (92-99\% yields and up to $97.5: 2.5$ er). It is worth noting that, owing to the mild reaction conditions and the avoidance of organometallic reagents that are usually utilized in cobalt-catalyzed cross-coupling reactions, impressive tolerance of functional groups on both reaction components (ester groups, cyano groups, aldehydes, siloxy groups, olefins, halides, etc.) was observed.

Reductive couplings for the AMPC-enabled radical DKR. Inspired by the above success, we further successfully developed a reductive cross-coupling process, which demonstrated the generality of the synergistic cobalt-photoredox catalysis strategy on asymmetric radical DKRs. Through a simple optimization of conditions (see the details in Supplementary Information), the replacement of DHP 2a with more easily available benzyl chloride as the alkyl source and Hantzsch ester (HE) as the reducing reagents facilely gives the same coupling product $\mathbf{3 a}$ in good yield and high enantioselectivity. Next, we probed the generality of this radical DKR via reductive coupling. As highlighted in Fig. 5, similar reaction efficiency and selectivity to the above transformation were observed in most cases (27 examples, $45-86 \%$ yields, up to 97.5:2.5 er). More significantly, linear alkyl iodine can participate in this reductive coupling provide alkyl-substituted chiral heterobiaryl product in good enantioselectivity that is inaccessible from corresponding DHP reagent, albeit in a moderate yield (3ba: $45 \%$ yield, 89.5:10.5 er).

Demonstration of the synthetic utility of this methodology. As privileged scaffolds, axially chiral heterobiaryls are widely used in asymmetric catalysis ${ }^{36}$. We supposed that the new chiral heterobiaryl molecules achieved through this radical DKR could be used as platforms for developing new axially chiral catalysts or ligands with isoquinoline as the hydrogen bond acceptor or the metal coordination site. Then, we treated chiral heterobiaryl 

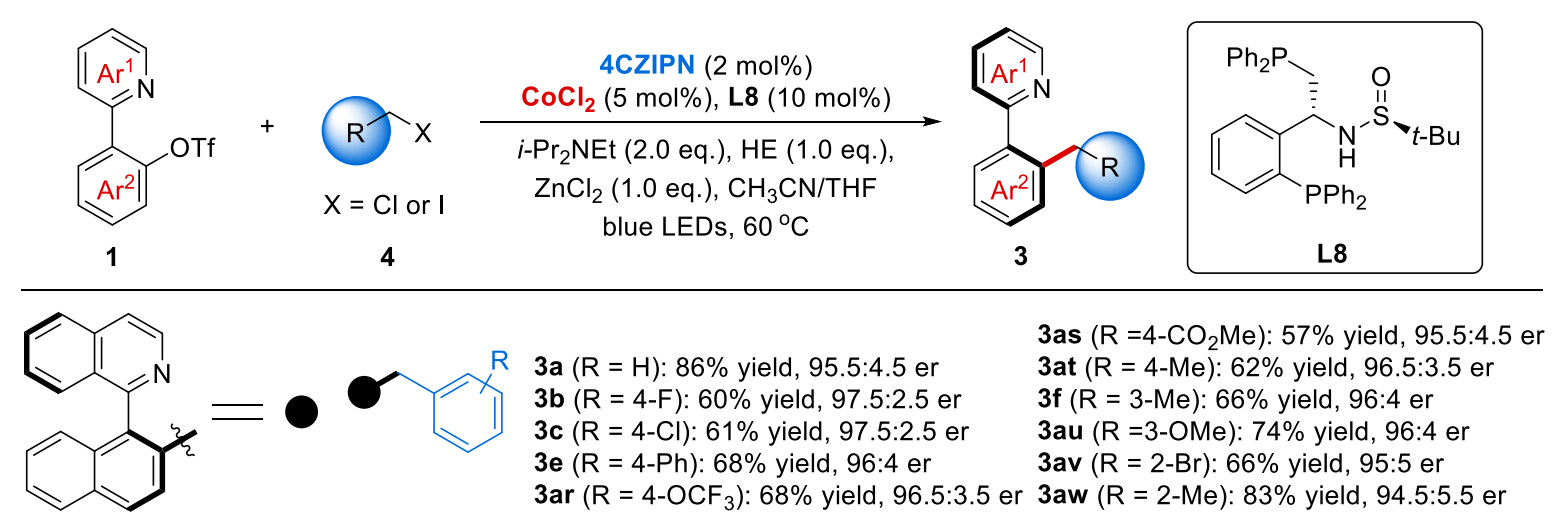

3a $(\mathrm{R}=\mathrm{H}): 86 \%$ yield, $95.5: 4.5 \mathrm{er}$ 3b $(R=4-F): 60 \%$ yield, $97.5: 2.5$ er $3 c(R=4-C l): 61 \%$ yield, $97.5: 2.5$ er 3e $(R=4-P h): 68 \%$ yield, $96: 4$ er 3as $\left(\mathrm{R}=4-\mathrm{CO}_{2} \mathrm{Me}\right): 57 \%$ yield, $95.5: 4.5 \mathrm{er}$ 3at $(R=4-M e): 62 \%$ yield, $96.5: 3.5$ er 3f $(\mathrm{R}=3-\mathrm{Me}): 66 \%$ yield, $96: 4$ er 3au ( $R=3-\mathrm{OMe}): 74 \%$ yield, $96: 4$ er 3av $(\mathrm{R}=2-\mathrm{Br}): 66 \%$ yield, $95: 5 \mathrm{er}$ 3ar $\left(\mathrm{R}=4-\mathrm{OCF}_{3}\right): 68 \%$ yield, $96.5: 3.5$ er 3 aw $(\mathrm{R}=2-\mathrm{Me}): 83 \%$ yield, $94.5: 5.5 \mathrm{er}$

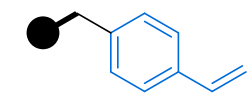

3ax:

$71 \%$ yield, $97.5: 2.5 \mathrm{er}$

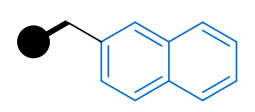

3ay: $56 \%$ yield, $97.5: 2.5$ er

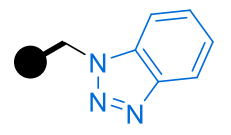

3az:

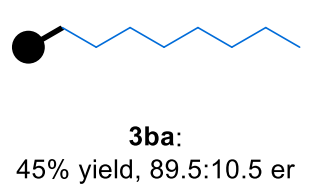

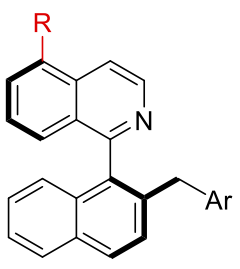

3bb : 70\% yield, 93.5:6.5 er<smiles>N#CCc1cccnc1-c1c(CCBr)ccc2ccccc12</smiles>

3bi:

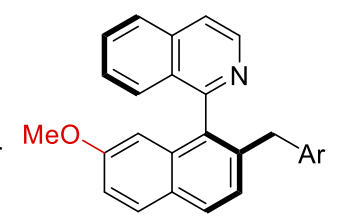

3bj:

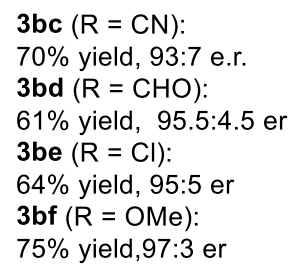

3 bc $(\mathrm{R}=\mathrm{CN})$ :

$70 \%$ yield, $93: 7$ e.r. 3bd $(\mathrm{R}=\mathrm{CHO})$ : $61 \%$ yield, $95.5: 4.5 \mathrm{er}$ 3be $(\mathrm{R}=\mathrm{Cl})$ : $64 \%$ yield, $95: 5$ er 3bf (R = OMe): $75 \%$ yield, $97: 3$ er<smiles>COc1cc(CCC2CCCCC2)c(-c2nccc3ccccc23)c2ccccc12</smiles>

3bk:
$46 \%$ yield, $95.5: 4.5$ er
$45 \%$ yield, 89.5:10.5 er

$65 \%$ yield, $96: 4$

$83 \%$ yield, $96.5: 3.5$ er $\quad 65 \%$ yield, $96.5: 3.5$ er

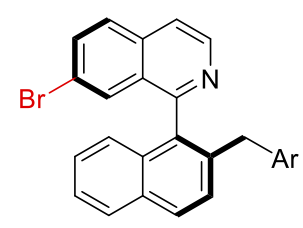

3bg: $65 \%$ yield, $95: 5$ er

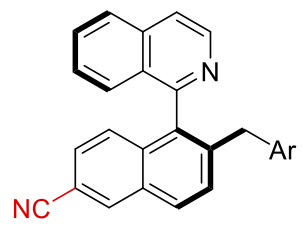

3bl:

$72 \%$ yield, $95: 5$ er

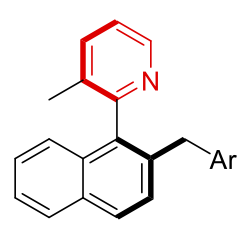

3bh:70\% yield, 96:4 er

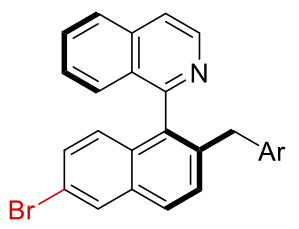

3bm:

$69 \%$ yield, $95.5: 4.5$ er

Fig. 5 | Substrate scope of AMPC-enabled radical DKR via reductive coupling. The optimal conditions: 1a (0.2 mmol), 4 (0.5 mmol), 4CzIPN ( $2 \mathrm{~mol} \%), \mathrm{CoCl}_{2}(5 \mathrm{~mol} \%), \mathbf{L 8}$ (10 mol\%), $\mathrm{ZnCl}_{2}$ (1.0 eq.), $i$ - $\mathrm{Pr}_{2} \mathrm{NEt}$ (2.0 eq.), $\mathrm{HE}$ (1.0 eq.) in $\mathrm{CH}_{3} \mathrm{CN} / \mathrm{THF}(1: 1,4.0 \mathrm{~mL}), 60{ }^{\circ} \mathrm{C}$. All the yields are isolated yields, and all er values were determined by chiral $\mathrm{HPLC}$ analysis. $\mathrm{Ar}=3-\mathrm{MeO}-\mathrm{C}_{6} \mathrm{H}_{4}$.

3aq with hydrazine hydrate in ethanol giving chiral primary amine $\mathbf{5}$ in $95 \%$ yield with conserved optical purity. Subsequently, condensation of this amine with isothiocyanate 10, aldehydes 11 and acid $\mathbf{1 2}$ and $\mathbf{1 3}$ produced potential bifunctional thiourea catalyst $\mathbf{6}$ and polydentate ligands 7-9 in good yields, respectively (Fig. 6a, i iv: 70-90\% yields). Application studies of these compounds are ongoing in our laboratory.

The aggregation-induced emission (AIE) caused by hindered intramolecular movement is one of the most popular design strategies for luminescence materials. In this technique, axially chiral compounds are often used, mostly by linking the inherent axially chiral skeleton with the luminescent group ${ }^{39}$. Here, we find a significant $\pi-\pi$ stacking effect (approximately $3.5 \AA$ A between the aryl ring of the side chain and isoquinoline by analyzing the crystal structure of chiral heterobiaryl product $\mathbf{3 h}$. According to this finding, we speculate that a donor-acceptor-type structure would make this type of axially chiral molecules a new class of AIE molecules with a different mechanism of action. To verify this conjecture, $\mathbf{3 k}$, which contains a good electron-donating carbazole group, was chosen as the platform molecule for 
a

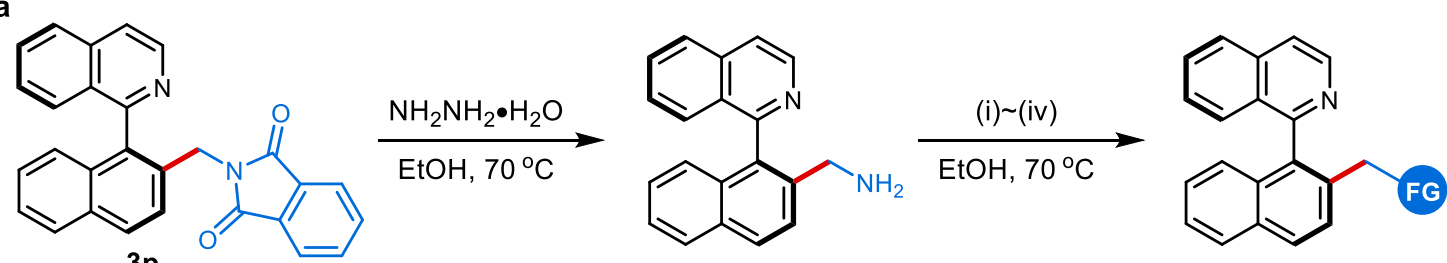
gram-scale: $1.14 \mathrm{~g}, 92 \%$ yield, $96: 4 \mathrm{er}$ 5: $94 \%$ yield, $96: 4$ er chiral catalysts or ligands 6-9
FG $=$ 6: $_{64 \% \text { yield }}^{\text {(i) }}$

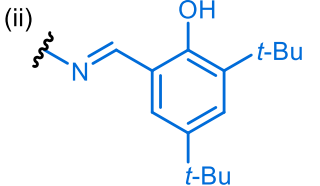
7: $70 \%$ yield

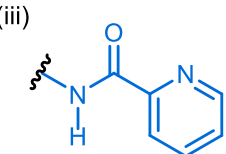
8: $90 \%$ yield

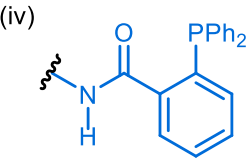
9: $89 \%$ yield

(i) 3,5-bis (trifluoromethyl) phenyl isothiocyanate 10 (1.0 eq.), DCM, rt; (ii), 3,5-di-tert-butylsalicylaldehyde 11 (1.0 eq.), $\mathrm{MgSO}_{4}$ (2.0 eq.), DCM, rt; (iii) 2-picolinic acid 12 (1.,2 eq.), EDCl (1.2 eq.), HOBT (1.2 eq.), i-Pr $\mathrm{Pr}_{2} \mathrm{NEt}$ (2.0 eq.), DCM, rt; (iv), 2(diphen-ylphosphino)benzoic acid 13 (1.0 eq.), DCC (1.2 eq.), DMAP (5 mol\%), DCM, rt.

b

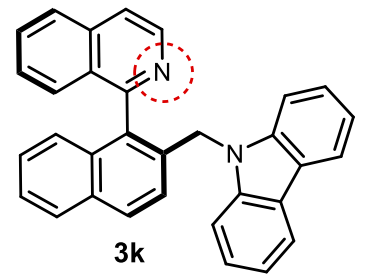

gram-scale: $1.26 \mathrm{~g}, 96 \%$ yield, $95: 5 \mathrm{er}$
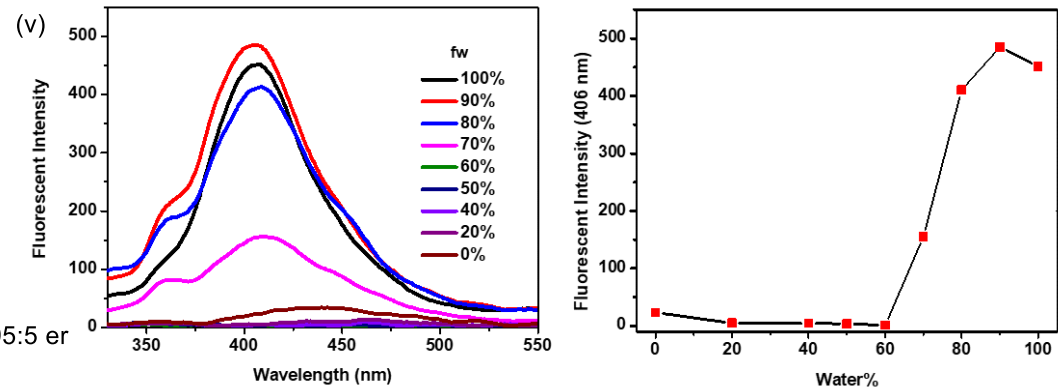

(vi)

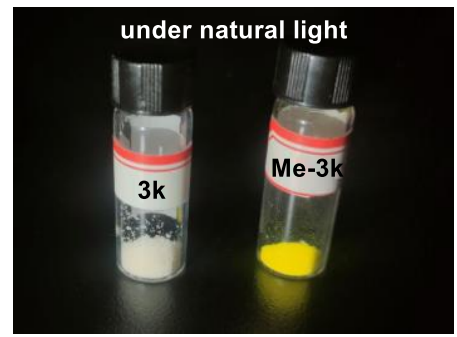

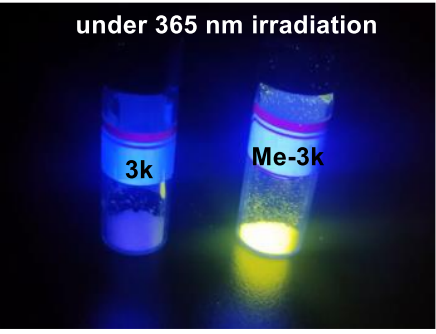
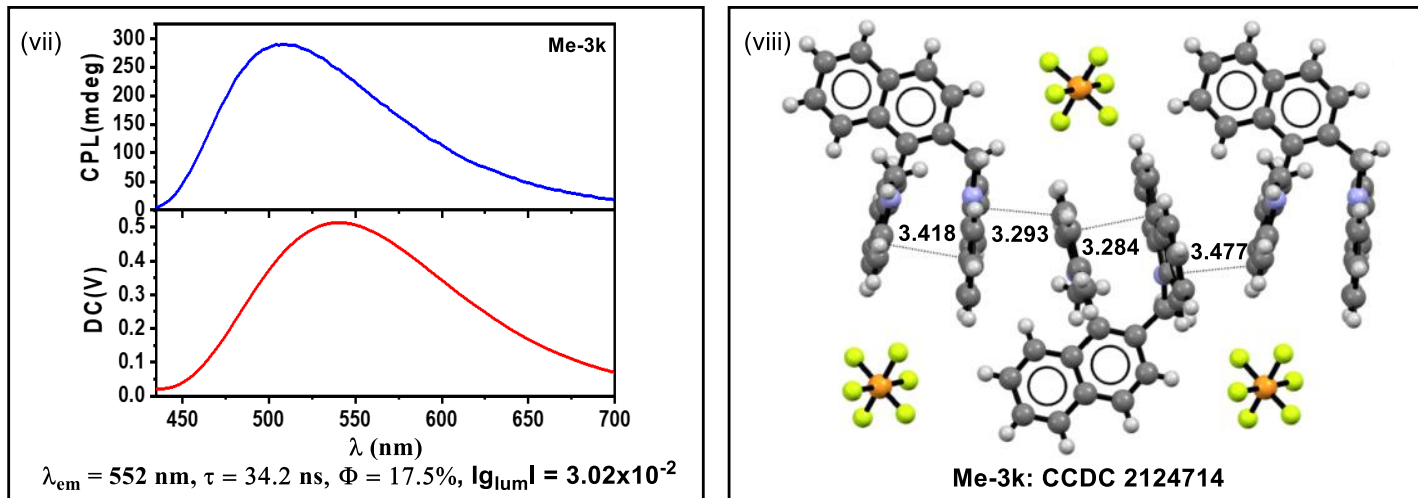

Fig. 6 | Demonstrations of the utilities of new methodology. (a) Application in the synthesis of axially chiral catalysts and ligands. (b) Application in the development of luminescent materials: (v) AIE experiments with 3k; (vi) photoluminescence of 3k and Me-3k; (vii) the luminous properties of Me-3k; (viii) single-crystal structure of Me-3k 
the AIE experiments. We found that $\mathbf{3 k}$ exhibited almost no fluorescence when dissolved in an acetonitrile solution, but as the proportion of water in the system increased, the emitted light intensity continued to increase, showing an obvious AIE effect (Fig. 6b, v). Subsequently, to reduce the band gap of the D-A structure, 3k was converted into the more electron-deficient quinolinium salt $\mathbf{M e - 3 k}$ by a routine methylation procedure involving isoquinoline (Fig. 6b, vi). The emission wavelength of this salt was obviously redshifted to the yellow region, and the powder exhibited strong fluorescence under $365 \mathrm{~nm}$ light irradiation. More significantly, a Me-3k film also showed a strong circularly polarized luminescence signal $\left(|\mathrm{glum}|=3.02 \times 10^{-2}\right.$; Fig. $6 \mathrm{~b}$, vii), which is difficult to achieve with axially chiral light-emitting organic small molecules ${ }^{40}$. Through the crystal analysis of Me-3k, we also found an obvious intra- and intermolecular $\pi$ - $\pi$ stacking effect between the aromatic rings (Fig. 6b, viii). Therefore, a layered structure was formed, and the naphthyl group on the axially chiral framework was spaced from the hexafluorophosphonate group to form a side wall. This highly ordered structure may be the cause of the highly circularly polarized signal.

Mechanistic considerations of the AESC-enabled DKR reaction. Next, we performed mechanistic studies to understand the radical DKR process. First, a set of control experiments was carried out (see Table S9 in the Supplementary Information), and the results confirmed that 1) visible light and the cobalt catalyst are indispensable for this reaction, 2) the omitting of the photocatalyst results in a much lower yield (27\% yield), albeit with a similar enantioselectivity, and 3) the addition of $i$ - $\operatorname{Pr}_{2} \mathrm{NEt}$ increases the yield by efficiently trapping the $\mathrm{TfOH}$ byproduct rather than by serving as an electron donor. The last conclusion was supported by the two control experiments shown in Fig. 7a, where the replacement of DHP reagent 2a with Me-2a yielded nearly the same results in the absence of $i$-Pr ${ }_{2} \mathrm{NEt}$. Next, stoichiometric experiments were performed by treating DHP reagent $\mathbf{M e - 2 a}, \mathrm{CoCl}_{2}$ and chiral polydentate ligand $\mathbf{L 8}$ under photocatalytic reduction conditions. As indicated in Fig. 7b, the chiral $\mathrm{Co}(\mathrm{I}) / \mathbf{L 8}$ complex, a proposed initial catalytic species in this asymmetric radical coupling, was detected by HRMS. By analyzing the redox potential of the Co(II)/L8 complex (Fig. 7e, P1, $\mathrm{E}_{1 / 2}{ }^{\text {red }}\left(\left[\mathrm{Co}^{\mathrm{II}} / \mathbf{L 8}\right] /\left[\mathrm{Co}^{\mathrm{I}} / \mathbf{L} 8\right]\right)=-1.07 \mathrm{~V}$ vs. SCE in $\left.\mathrm{MeCN} / \mathrm{THF}\right)$, the initial $\mathrm{Co}(\mathrm{I}) / \mathbf{L 8}$ species was generated through reduction by the reduced state of the $4 \mathrm{CzIPN}$ photocatalyst $\left(\mathrm{E}_{1 / 2}{ }^{\text {red }}\left(4 \mathrm{CzIPN} / 4 \mathrm{CzIPN}^{-}\right)=-1.24 \mathrm{~V} \text { vs. SCE in } \mathrm{MeCN}\right)^{41}$ or the excited state

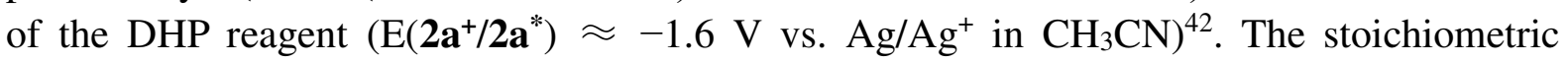
oxidative addition process with $\mathrm{Co}(\mathrm{I})\left(\mathrm{PPh}_{3}\right)_{3} \mathrm{Cl}$, ligand $\mathbf{L 8}$ and heterobiaryl substrate $\mathbf{1 a}$ was implemented, and the proposed Co(III) intermediate was also detected by HRMS (Fig. 7c). Although we failed to obtain single crystals of chiral cobalt complexes through long-term effort, we confirmed that this radical DKR process was catalyzed by the cobalt/L8 complex in a 1:1 ratio according to the results of nonlinear experiments (Fig. 7d) and HRMS analysis (Fig. 7b and 7c).

\section{Conclusion}

We have developed a metallaphotoredox-catalyzed DKR process of racemic heterobiaryls under visible light irradiation. The identification of a chiral polydentate ligand for cobalt-catalyzed enantioselective radical couplings was critical to the successful implementation of this research. As a result, a variety of chiral heterobiaryl products were afforded with generally high efficiency and selectivity. In addition, this free radical DKR 
<smiles>[O+]c1cccc2ccnc(-c3cccc4ccccc34)c12</smiles>

1a<smiles>[R]N1C(C)=C(C(=O)OCC)C(Cc2ccccc2)C(C(=O)OCC)=C1C</smiles>

2a $(\mathrm{R}=\mathrm{H})$

$N$-Me-2a $(\mathrm{R}=\mathrm{Me})$

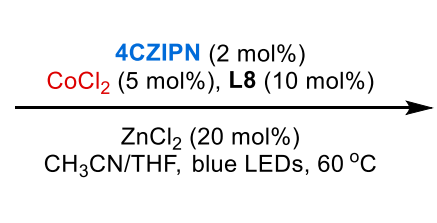

$\mathrm{ZnCl}(20 \mathrm{~mol} \%)$
$\mathrm{CH}_{3} \mathrm{CN} / \mathrm{THF}$, blue LEDs, $60^{\circ} \mathrm{C}$

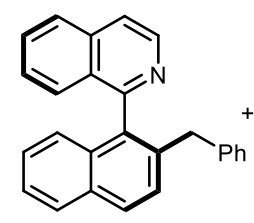

3aa<smiles>CCOC(=O)c1cc(C(=O)OCC)c(Br)[n+](C)c1C</smiles>

byproduct

Using 2a and $i-\mathrm{Pr}_{2} \mathrm{NEt}$ (1.0 eq.): $92 \%$ GC yield, 96:4 er; using 2a only: 33\% GC yield, $96: 4$ er

Using $\mathbf{N}$-Me-2a and $i-\mathrm{Pr}_{2} \mathrm{NEt}$ (1.0 eq.): $92 \%$ GC yield, $97: 3$ er; using $\mathbf{N}-\mathbf{M e}-\mathbf{2 a}$ only: $95 \%$ GC yield, $97: 3$ er

b<smiles>CCOC(=O)C1=C(C)N(C)C(C)=C(C(=O)OCC)C1Cc1ccccc1</smiles>

N-Me-2a $(0.15 \mathrm{mmol})$

$0.1 \mathrm{mmol}$<smiles>CC(C)(C)S(=O)N(c1ccccc1)[C@@H](CPc1ccccc1)c1ccccc1</smiles>

L8 $(0.1 \mathrm{mmol})$

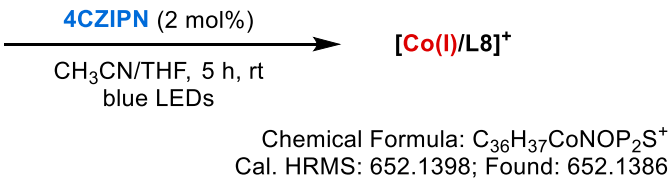

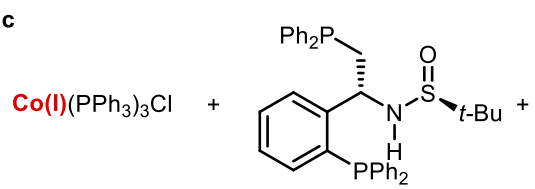

$0.1 \mathrm{mmol}$

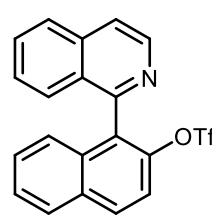

1a $(0.11 \mathrm{mmol})$
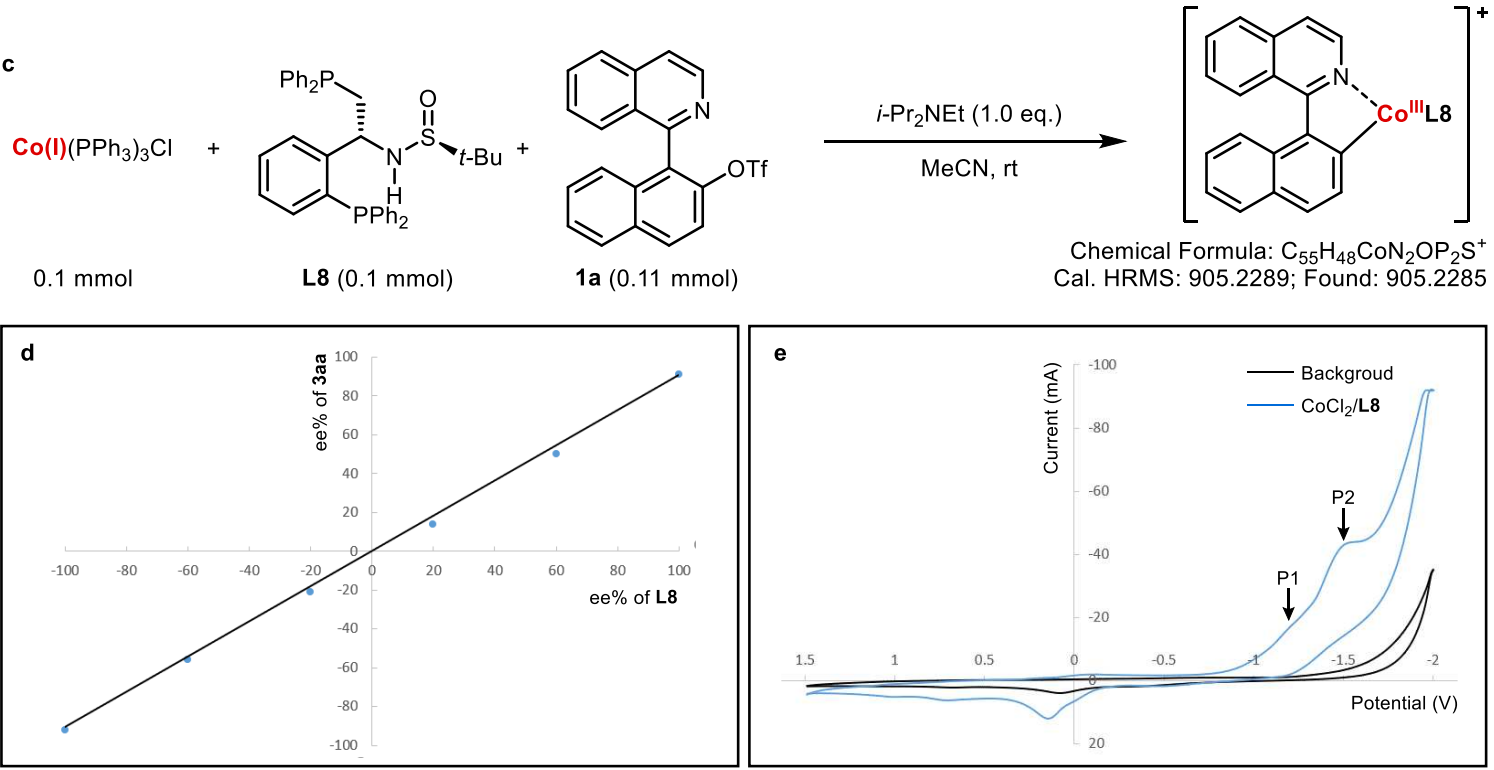

Fig. 7 | Mechanistical investigations. a, Confirmation of the $i$ - $\operatorname{Pr}_{2} \mathrm{NEt}$ additive as the acid capturer. b-c, Determination of the proposed transient $\mathrm{Co}(\mathrm{I})$ and $\mathrm{Co}(\mathrm{III})$ species. $\mathrm{d}$, Nonlinear effect experiments with the model reaction. e, Cyclic voltammetry experiment with the $\mathrm{CoCl}_{2} / \mathbf{L 8}$ (1:1) complex.

process has been proved to have a certain universality and can be applied to reductive coupling reactions. The value of this methodology was further proven by converting these new axially chiral heterobiaryl molecules to promising chiral catalysts and ligands, as well as by finding new chiral fluorescent materials. We believed that, this research not only opens a new window for asymmetric photochemical synthesis under visible light and cobalt-catalyzed asymmetric radical couplings but also creates an avenue to significant chiral heterobiaryl scaffolds and related functional materials.

\section{Methods}

General procedure for the AMPC-enabled DKR reaction. In an argon-filled glove box, a $10 \mathrm{~mL}$ vial equipped with a magnetic stirrer bar was charged sequentially with $\mathbf{L 8}(0.02 \mathrm{mmol}, 10 \mathrm{~mol} \%)$ and $\mathrm{CoCl}_{2}(0.01 \mathrm{mmol}, 5 \mathrm{~mol} \%)$, followed by the addition of THF $(2 \mathrm{~mL})$ and $\mathrm{MeCN}(2 \mathrm{~mL})$. After stirring at room temperature for $30 \mathrm{~min}$, substrates $\mathbf{1 a}(0.2 \mathrm{mmol})$ and $\mathbf{2 a}(0.3 \mathrm{mmol})$, photocatalyst 
4CzIPN ( $2 \mathrm{~mol} \%, 0.004 \mathrm{mmol}$ ), $i$ - $\mathrm{Pr}_{2} \mathrm{NEt}$ (1.0 eq., $0.2 \mathrm{mmol}$ ) and $\mathrm{ZnCl}_{2}(20 \mathrm{~mol} \%, 0.04 \mathrm{mmol}$ ) were added to the resulting mixture. Then, the mixture was stirred at a distance of $\sim 10 \mathrm{~cm}$ from $90 \mathrm{~W}$ blue LEDs at $60{ }^{\circ} \mathrm{C}$ for approximately $10 \mathrm{~h}$ until the reaction was completed, as monitored by TLC analysis. The concentrated reaction residue was purified by flash column chromatography on silica gel to afford desired product $\mathbf{3 a}$.

\section{Data availability}

Crystallographic data for the structures reported in this paper have been deposited at the Cambridge Crystallographic Data Centre under deposition numbers CCDC 2124717 (3h) and 2124714 (Me-3k). Copies of the data can be obtained free of charge via https://www.ccdc.cam.ac.uk/structures/. All other characterization data are in the supplementary materials. All data are available in the main text or the supplementary materials.

\section{References}

1. Schultz, D. M. \& Yoon, T. P. Solar Synthesis: Prospects in visible light photocatalysis. Science 343, 1239176 (2014).

2. Marzo, L., Pagire, S. K., Reiser, O. \& König, B. Visible-light photocatalysis: does it make a difference in organic synthesis? Angew. Chem. Int. Ed. 57, 10034-10072 (2018).

3. Brimioulle, R., Lenhart, D., Maturi, M. M. \& Bach, T. Enantioselective catalysis of photochemical reactions. Angew. Chem. Int. Ed. 54, 3872-3890 (2015).

4. Silvil, M. \& Melchiorre, P. Enhancing the potential of enantioselective organocatalysis with light. Nature 554, 41-49 (2018).

5. Lipp, A., Badir, S. O. \& Molander, G. A. Stereoinduction in metallaphotoredox catalysis. Angew. Chem. Int. Ed. 60, 1714-1726 (2021).

6. Schmermund, L. et al. Photo-biocatalysis: biotransformations in the presence of light. ACS Catal. 9, 4115-4144 (2019).

7. Bauer, A., Westkämper, F., Grimme, S. \& Bach, T. Catalytic enantioselective reactions driven by photoinduced electron transfer. Nature 436, 1139-1140 (2005).

8. Nicewicz, D. A. \& MacMillan, D. W. C. Merging photoredox catalysis with organocatalysis: the direct asymmetric alkylation of aldehydes. Science 322, 77-80 (2008).

9. Arceo, E., Jurberg, I. D., Álvarez-Fernaández, A. \& Melchiorre, P. Photochemical activity of a key donor-acceptor complex can drive stereoselective catalytic a-alkylation of aldehydes. Nat. Chem. 5, 750-756 (2013).

10. Tellis, J. C., Primer, D. N. \& Molander, G. A. Single-electron transmetalation in organoboron cross-coupling by photoredox/nickel dual catalysis. Science 345, 433-436 (2014).

11. Du, J., Skubi, K. L., Schultz, D. M. \& Yoon, T. P. A dual-catalysis approach to enantioselective [2+2] photocycloadditions using visible light. Science 344, 392-396 (2014).

12. Huo, H. et al. Asymmetric photoredox transition-metal catalysis activated by visible light. Nature 515, 100-103 (2014).

13. Kainz, Q. M. et al. Asymmetric copper-catalyzed C-N cross-couplings induced by visible light. Science 351, 681-684 (2016).

14. Emmanuel, M. A., Greenberg, N. R., Oblinsky, D. G. \& Hyster, T. K. Accessing non-natural reactivity by irradiating nicotinamide-dependent enzymes with light. Nature 540, 414-417 (2016). 
15. Litman, Z. C., Wang, Y.-J., Zhao, H.-M. \& Hartwig, J. F. Cooperative asymmetric reactions combining photocatalysis and enzymatic catalysis. Nature 560, 355-359 (2018).

16. Shin, N. Y., Ryss, J. M., Zhang, X., Miller, S. J. \& Knowles, R. R. Light-driven deracemization enabled by excited-state electron transfer. Science 366, 364-369 (2019).

17. Cheng, J. K., Xiang, S.-H., Li, S., Ye, L. \& Tan, B. Recent advances in catalytic asymmetric construction of atropisomers. Chem. Rev. 121, 4805-4902 (2021).

18. Hölzl-Hobmeier, A. et al. Catalytic deracemization of chiral allenes by sensitized excitation with visible light. Nature 564, 240-243 (2018).

19. Plaza, M., Großkopf, J., Breitenlechner, S., Bannwarth, C. \& Bach, T. Photochemical deracemization of primary allene amides by triplet energy transfer: a combined synthetic and theoretical study. J. Am. Chem. Soc. 143, 11209-11217 (2021).

20. Hapke, M. \& Hilt, G. (eds) Cobalt Catalysis in Organic Synthesis (Wiley-VCH, 2020).

21. Mao, J. et al. Cobalt-bisoxazoline-catalyzed asymmetric Kumada cross-coupling of racemic $\alpha$-bromo esters with aryl grignard reagents. J. Am. Chem. Soc. 136, 17662-17668 (2014).

22. $\mathrm{Li}, \mathrm{Z}$. et al. A cobalt-catalyzed enantioconvergent radical Negishi $\mathrm{C}\left(\mathrm{sp}^{3}\right)-\mathrm{C}\left(\mathrm{sp}^{2}\right)$ cross-coupling with chiral multidentate N,N,P ligand. Organometallics 40, 2215-2219 (2021).

23. Li, Y. et al. Cobalt-catalysed enantioselective $\mathrm{C}\left(\mathrm{sp}^{3}\right)-\mathrm{C}\left(\mathrm{sp}^{3}\right)$ coupling. Nat. Cat. 4, 901-911 (2021).

24. Fu, G. C. Transition-metal catalysis of nucleophilic substitution reactions: a radical alternative to $\mathrm{S}_{\mathrm{N}} 1$ and $\mathrm{S}_{\mathrm{N}} 2$ processes. ACS Cent. Sci. 3, 692-700 (2017).

25. Wang, F., Chen, P. \& Liu, G. Copper-catalyzed radical relay for asymmetric radical transformations. Acc. Chem. Res. 51, 2036-2046 (2018).

26. Gu, Q.-S., Li, Z.-L. \& Liu, X.-Y. Copper(I)-catalyzed asymmetric reactions involving radicals. Acc. Chem. Res. 53, 170-181 (2020).

27. Twilton, J. et al. The merger of transition metal and photocatalysis. Nat. Rev. Chem. 1, 0052 (2017).

28. Kojima, M. \& Matsunaga, S. The merger of photoredox and cobalt catalysis. Trends Chem. 2, 410-426 (2020).

29. Ding, W. et al. Bifunctional photocatalysts for enantioselective aerobic oxidation of $\beta$-ketoesters. J. Am. Chem. Soc. 139, 63-66 (2017).

30. Lu, F.-D. et al. Asymmetric propargylic radical cyanation enabled by dual organophotoredox and copper catalysis. J. Am. Chem. Soc. 141, 6167-6172 (2019).

31. Chen, J. et al. Photoinduced copper-catalyzed asymmetric C-O cross-coupling. J. Am. Chem. Soc. 143, 13382-13392 (2021).

32. Kakiuchi, F., Gendre, P. L., Yamada, A., Ohtaki, H. \& Murai, S. Atropselective alkylation of biaryl compounds by means of transition metal-catalyzed $\mathrm{C}-\mathrm{H} /$ olefin coupling. Tetrahedron: Asymmetry 11, 2647-265 (2000).

33. Ros, A. et al. Dynamic kinetic cross-coupling strategy for the asymmetric synthesis of axially chiral heterobiaryls. J. Am. Chem. Soc. 135, 15730-15733 (2013).

34. Bhat, V., Wang, S., Stoltz, B. M. \& Virgil, S. C. Asymmetric synthesis of QUINAP via dynamic kinetic resolution. J. Am. Chem. Soc. 135, 16829-16832 (2013).

35. Wang, Q., Cai, Z.-J., Liu, C.-X., Gu, Q. \& You, S.-L. Rhodium-catalyzed atroposelective C-H arylation: efficient synthesis of axially chiral heterobiaryls. J. Am. Chem. Soc. 141, 9504-9510 (2019). 
36. Carmona, J. A., Rodríguez-Franco, C., Fernández, R., Hornillos, V. \& Lassaletta, J. M. Atroposelective transformation of axially chiral (hetero)biaryls. From desymmetrization to modern resolution strategies. Chem. Soc. Rev. 50, 2968-2983 (2021).

37. Jin, M.-Y. \& Yoshikai, N. Cobalt-xantphos-catalyzed, LiCl-mediated preparation of arylzinc reagents from aryl iodides, bromides, and chlorides. J. Org. Chem. 76, 1972-1978 (2011).

38. Zhou, W. et al. Chiral sulfinamide bisphosphine catalysts: design, synthesis, and applicationin highly enantioselective intermolecular cross-Rauhut-Currier reactions. Angew. Chem. Int. Ed. 54, 14853-14857 (2015).

39. Hu, M., Feng, H.-T., Yuan, Y.-X., Zheng, Y.-S. \& Tang, B. Z. Chiral AIEgens-chiral recognition, CPL materials and other chiral applications. Coordin. Chem. Rev. 416, 213329 (2020).

40. Han, J. et al. Enhanced circularly polarized luminescence in emissive charge-transfer complexes. Angew. Chem. Int. Ed. 58, 7013-7019 (2019).

41. Speckmeier, E., Fischer, T. G. \& Zeitler K. A toolbox approach to construct broadly applicable metal-free catalysts for photoredox chemistry: deliberate tuning of redox potentials and importance of halogens in donor-acceptor cyanoarenes. J. Am. Chem. Soc. 140, 15353-15365 (2018).

42. Gandolfo, E., Tang, X., Roy, S. R. \& Melchiorre, P. Photochemical asymmetric nickel-catalyzed acyl cross-coupling. Angew. Chem. Int. Ed. 58, 16854-168587 (2019).

\section{Acknowledgements}

Financial support from the National Natural Science Foundation of China (No. 91956201, 21820102003, 21822103, 21772052 and 21772053), the Natural Science Foundation of Hubei Province (2017AHB047) and the International Joint Research Center for Intelligent Biosensing Technology and Health are acknowledged. We thank Prof. Ming-Yuan Liu for the determination of circularly polarized luminescence signal $\left(\left|\mathrm{g}_{\mathrm{lum}}\right|\right)$ and Prof. Fang-Fang Pan for the X-ray single crystal diffraction analysis. We thank Prof. Jia-Rong Chen and Associate Prof. Ying Cheng for helpful discussions.

\section{Author contributions}

L.Q.L. and W.J.X. directed the project and wrote the manuscript with input from all other authors. Under the guidance of L.Q.L. and W.J.X., X.J. and W.X. developed the methods and designed and performed the synthetic and mechanistic experiments with the help of F.D.L, Y.J., Q.Y., L.Y.X. In addition, S.D. performed the DFT calculations under the guidance of X.Q. and J.A.T. gave many helpful suggestions and helped to revise this manuscript. All the authors participated in the discussion and preparation of the manuscript.

\section{Competing interests}

The authors declare no competing interests.

\section{Additional information}

Supplementary information The online version contains supplementary material available at https://doi.org/10.1038/s41929-xxx-xxxxx-x.

Correspondence and requests for materials should be addressed to Liang-Qiu Lu or Wen-Jing Xiao.

Peer review information Nature Catalysis thanks xxx and the other, anonymous, reviewer(s) for their 
contribution to the peer review of this work.

Reprints and permissions information is available at www.nature.com/reprints.

Publisher's note Springer Nature remains neutral with regard to jurisdictional claims in published maps and institutional affiliations. 


\section{Supplementary Files}

This is a list of supplementary files associated with this preprint. Click to download.

- SupplementaryMaterials.pdf 